\title{
Extruded Plastic Scintillator for MINERvA
}

\author{
Anna Pla-Dalmau, Alan D. Bross, Victor V. Rykalin and Brian M. Wood
}

\begin{abstract}
An extrusion line has recently been installed at Fermilab in collaboration with NICADD (Northern Illinois Center for Accelerator and Detector Development). This new facility will serve to further develop and improve extruded plastic scintillator. Since polystyrene is widely used in the consumer industry, the logical path was to investigate the extrusion of commercial-grade polystyrene pellets with dopants to yield high quality plastic scintillator. The DO and MINOS experiments are already using extruded scintillator strips in their detectors. A new experiment at Fermilab is pursuing the use of extruded plastic scintillator. A new plastic scintillator strip is being tested and its properties characterized. The initial results are presented here.
\end{abstract}

\section{INTRODUCTION}

$\mathrm{M}$ INERVA, which is planned to run in the NuMI Beamline at Fermilab, is an experiment to study neutrino interactions in a fine-grained detector. Because of the high intensity of the NuMI neutrino beam, high-statistics neutrino measurements can be carried out with a totally active target made of several tons of extruded plastic scintillator [1]. The FNAL-NICADD Extrusion Facility at Fermilab will prepare this scintillator $[2,3]$. The extruded scintillator for the Active Target will have a triangular shape $(33 \mathrm{~mm}$ wide and 17 $\mathrm{mm}$ high). The strip contains a hole centered along the vertical axis where a 1.2-mm diameter wavelength shifting (WLS) fiber will be inserted. The diameter of the hole is specified at $1.5 \pm 0.1 \mathrm{~mm}$.

Here we present the results of the attenuation lengths measurements performed, the effect of the hole diameter on light yield and the light yield determined using the cosmic ray test-stand.

\section{EXPERIMENTAL SECTION}

The FNAL/NICADD extrusion line has been operating on a commissioning and R\&D bases since April 2003. The polystyrene (PS) pellets used are Dow Styron $663(\mathrm{~W})$. This is a commercial grade, general-purpose polystyrene without additives. The dopants currently added are 1\% PPO and

This work was supported by the U.S. Department of Energy under Contract No. DE-AC02-76CHO3000 and in part by the U.S. Department of Education under Grant No. P116Z010035, the U.S. Department of Energy and the State of Illinois Higher Education Cooperation Act.

A. Pla-Dalmau and A. D. Bross are with Fermi National Accelerator Laboratory, Batavia, IL 60510 USA (e-mail: pla@fnal.gov, bross@fnal.gov).

V. V. Rykalin and B. M. Wood with the Northern Illinois Center for Accelerator and Detector Development (NICADD), Northern Illinois University, DeKalb, IL 60115 USA (e-mail: rykalin@fnal.gov).
$0.03 \%$ POPOP available in a pre-mixed form from Curtiss Laboratories (Bensalem, PA). This is a blue-emitting scintillator with an absorption cut-off at approximately $400 \mathrm{~nm}$ (for a 1-cm path length) and an emission maximum at $420 \mathrm{~nm}$.

\section{DISCUSSION}

\section{A. Attenuation Length Measurements}

In order to measure the attenuation length of the triangular strips we first selected forty different 1-m long strips; half of which had a hole that would fit a 1.2-mm WLS fiber (called the $1.2-\mathrm{mm}$ hole), and the other half with a hole that would suit a 1.5-mm diameter WLS fiber (called the 1.5-mm hole). We then polished one end of the strip, placed the strip in a Tyvek sock (one sock formed to slide on and off from each strip as to avoid irregularities in separate wrappings), and inserted it inside a black box with the polished end touching the PMT. A WLS fiber was not used in this setup. Then by measuring the light yield induced by a ${ }^{137} \mathrm{Cs}$ gamma source along a number of set points down the strip, we can compute the attenuation length by examining the exponential growth of the derived light yields. As expected, the attenuation lengths were independent of the hole sizes; the long and short components were $42.15 \pm 1.14 \mathrm{~cm}$ and $30.52 \pm 0.74 \mathrm{~cm}$ respectively for the $1.2-\mathrm{mm}$ hole, and $44.05 \pm 0.94 \mathrm{~cm}$ and $30.33 \pm 0.77 \mathrm{~cm}$ for the 1.5-mm hole. These give errors of 2-3\%, which makes for very homogenous results.

\section{B. Scintillation Light Yield}

The setup was similar to that of the attenuation length measurements, with a few changes to accommodate for the optical WLS fiber. We wound the fiber (about $1.5 \mathrm{~m}$ long) around into the photomultiplier tube, and used a fixture to hold it so there was direct contact with the surface of the PMT. We measured only four points along the strip because of the small change in light yield due to the long attenuation length of the WLS fiber (on the order of five meters). Then we took measurements of an assortment of different strips with varying hole diameters.

In order to measure if the size of the hole would affect the light yields of scintillator strips we measured a sample with a variety of hole sizes. Regardless of the hole size, we inserted the same 1.2-mm WLS fiber and measured the resultant light yield. Figure 1 shows the approximate ratios of hole size to fiber size, with 0.9 being a close fit and 0.3 being very loose. A total of 25 strips were measured. 


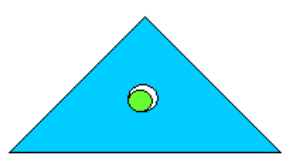

0.9

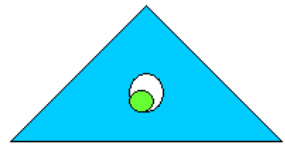

0.5

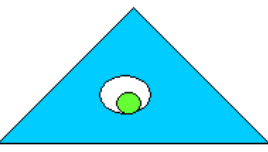

0.3
Fig. 1. Hole to fiber ratios.

The strips were measured with a reflective foil touching the far end of the WLS fiber, but in order to avoid irregularities in the contact with the fiber we also re-measured without foil. The average light yield of the scintillator strips with the reflective end compared to that of the strips without the foil was 1.41 times greater. Figure 2 shows that a better match between hole and fiber diameter (0.75-0.9 ratios) does not render a higher light yield. A large mismatch between hole and fiber diameter represented in the lower-ratio range results in light yields that are similar to the rest of the data. The variations in the measurements were larger when the light yield was measured without a foil at the farthest end of the scintillator strip. The spread of the measurements is approximately $9 \%$. The "Far Light Yield" and the "Near Light Yield" refer to the points measured farthest from and nearest to the PMT respectively. "Average Light Yield" is the mean of all four data points. No correlation is observed in the case either between the hole/fiber ratio and the light yield. Figures 2 illustrates that the hole size does not have a significant effect in the light yields.

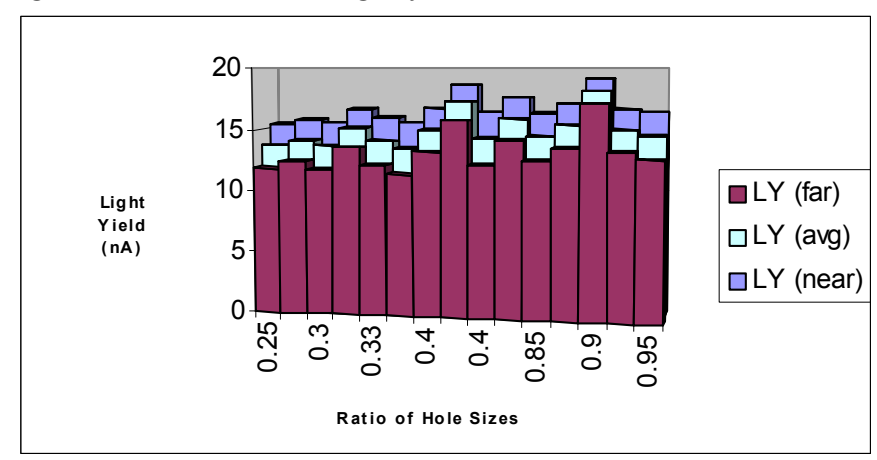

Fig. 2. Effect on Light Yield Due to Hole Sizes without Reflective End.

In preparation to check the glue effect in the light yield, we selected six fibers and six strips without the reflective foil. We measured each fiber in one strip of scintillator as a means of calibrating the fibers. After comparing the fibers, we then inserted each one into a designated scintillator strip. Each strip was selected to show the effects of a wide range of hole sizes. The approximate hole to fiber ratios were: $0.9,0.9,0.4,0.3$, 0.3 , and 0.3 . After normalizing the strips to the calibrated fiber data previously mentioned (as to ignore the effects of separate fibers), we again found that the size of the hole had no significant effect on the resultant light yields. We then filled the holes of the six scintillator strips with BC600 glue to examine how it would affect the results. Each fiber was kept in the same scintillator strip to avoid any inconsistencies. The light yields of the scintillator strips with the glued fiber were approximately 1.9-2.0 times higher that those without glue.

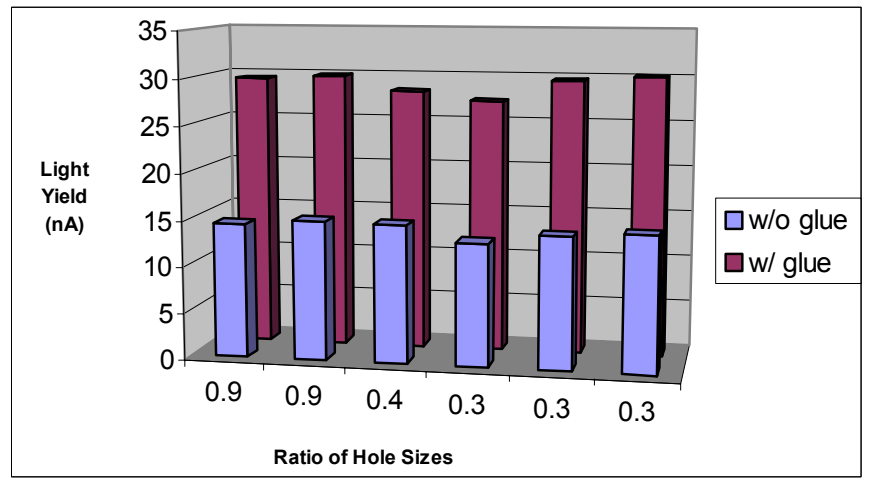

Fig. 3. Average Light Yield With and Without Glue.

\section{Cosmic Ray Test}

The last test performed on the triangular scintillator was to determine the light yield expressed as number of photoelectrons using cosmic rays. Two scintillator strips $(1 \mathrm{~m}$ long) individually wrapped in Tyvek and taped together were used. The WLS fibers were glued in the hole using BC600. The fibers were connected to a photomultiplier tube (PMT) which proceeded to a delay and finally to an analog-digital converter (ADC). The total length of WLS fibers was $1.5 \mathrm{~m}$. Hamamatsu H3178-61 PMT was used for these measurements. This PMT has a bialkali photocathode with peak wavelength sensitivity at $420 \mathrm{~nm}$. The size of the trigger counters was significantly less than size of one triangle side. The surface of each counter was about $15 \mathrm{~cm}^{2}$ which corresponds to $0.7 \%$ of the total surface of the side where the triggers were mounted. Both trigger counters were positioned in the middle of the scintillator strip and connected to an amplifier, discriminator, and coincidence unit, which subsequently linked to the ADC. All the components involving scintillator or photomultiplier tubes were placed inside a lightproof box.

The average number of photoelectrons, $\mathrm{N}$, can be estimated from the charge spectrum utilizing the following relationship:

$$
N=\left(\frac{A}{\sigma}\right)^{2}
$$

In this equation, $\mathrm{A}$ is the mean of the entire distribution and $\sigma$ is the standard deviation for the entire distribution [4]. The mean and the standard deviation can be derived from fitting the data of the charge spectrum to a Gauss distribution. The amplitude distribution and fit to the left edge of the distribution is shown on Fig. 4. The pedestal is in count number 49 of the ADC. In our measurements, $\mathrm{N}$ was $18.4 \pm 1.5$ photoelectrons for a one triangular strip normalized to the thickness of $1.7 \mathrm{~cm}$. The scaling to the first electron distribution gives approximately the same value. The result was consistent within $20 \%$ for the three measurements performed. The measurements were done without reflective end. The use of a reflective end will increase the light yield by a factor of 1.4 to 1.5 as mentioned in an earlier section. 


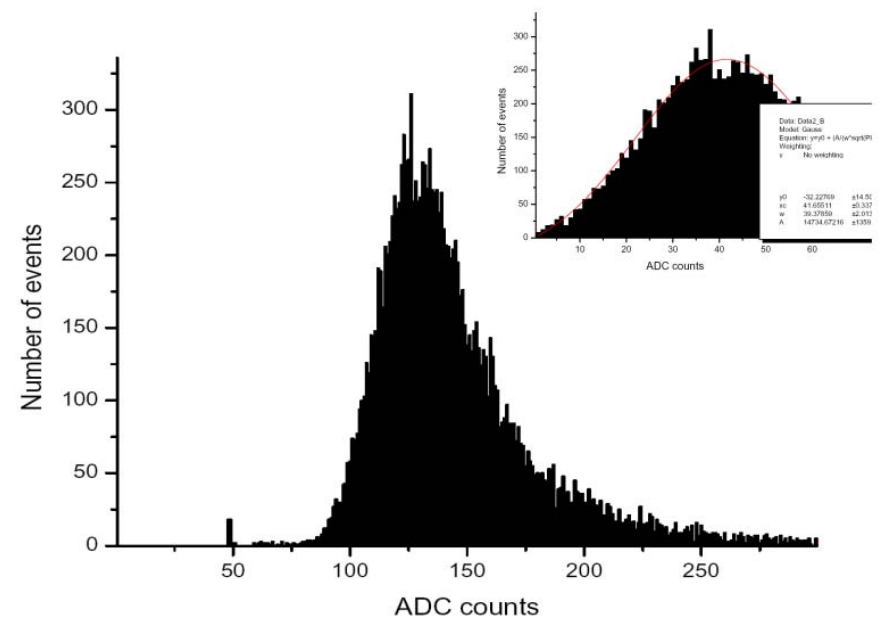

Fig. 2. Amplitude distribution.

\section{CONCLUSION}

R\&D work for MINERvA has started and will continue through 2005. The extruded plastic scintillator shows the high quality characteristics that we have measured in the past. The FNAL/NICADD approach with the in-line continuous extrusion equipment yields high quality scintillator and continues to offer the opportunity to see further improvement in attenuation length and light yield as more parameters are investigated.

\section{REFERENCES}

[1] Kevin McFarland, MINERvA, Introduction, Detector Progress, and MRI Proposal April 2004. 2-7

[2] O. A. Grachov, Study of New FNAL-NICADD Extruded Scintillator as Active Media of Large EMCAL of ALICE at LHC, May 2004. 2-3

[3] D. Besnosko, A. Bross, A. Pla-Dalmau, A. Dyshkant, V. Rykalin. FnalNicadd Extruded Scintillator, FERMILAB-CONF-04-216-E, Oct 2004. 4pp. (NSS / MIC), Rome, Italy, 16-22 Oct 2004

[4] http://dustbunny.physics.indiana.edu/ shepherd/cebaf/node33.html 